not attend any training programme. The post-tests were conducted on both groups to collect the data on the variables of the study. The data pertaining to health-related physical fitness components were analysed by paired ' $t$ ' test to determine the difference between initial and final mean for experimental and control groups. Significant difference seen at the 0.05 level with 29 degree of freedom is 2.045 and at 0.01 level with 29 degree of freedom is 2.756 in experimental group following 12 weeks of aerobic training programme for cardiorespiratory endurance, flexibility, muscular strength endurance and skin fold thickness (body fat \%). In the case of control group no significant changes were seen in any of the selected variables. The conclusions of this study are improved cardiorespiratory endurance, flexibility, muscular strength endurance and decreased skin fold thickness (body fat \%) among the experimental group of middle-aged women after 12 weeks of aerobic training.

\section{EFFECT OF AEROBIC EXERCISE PROGRAMME ON HEALTH RELATED PHYSICAL FITNESS COMPONENTS OF MIDDLE AGED WOMEN}

A Shahana, Usha S Nair, S S Hasrani Lakshmibai National College of Physical Education, Trivandrum, Kerala, India

\subsection{6/bjsm.2010.078725.60}

Fitness for living in the house or on the farm or at office or factory or in work places or in any service implies freedom from disease, enough strength, endurance and other abilities to meet the demands of daily living. Doing physical activity everyday contributes to optimum health and quality oflife. Life styles can be changed to improve health and fitness through daily exercises. Aerobic exercise stimulates heart, lungs and all working group of muscles and produces beneficial changes in body and mind. Many physiological changes are determined by daily aerobic exercises. The purpose of the study was to determine the effect of a 12-week aerobic exercise programme on healthrelated physical fitness components, which are cardiorespiratory endurance, flexibility, abdominal strength endurance and body fat in middle-aged women. A total of 60 middle-aged women from Karyavattom panchayath of Trivandrum district in Kerala state between the age group of 35 and 45 years were selected as subjects for the study. They were tested to collect the data on selected variables. The cardiorespiratory endurance, flexibility, abdominal strength endurance and body fat percentage were selected variables. Further, 30 subjects were randomly assigned as experimental group and 30 as control group. The experimental group underwent aerobic exercise training thrice a week for 12 weeks. The control group did 